\title{
Engineering computational method of process of fluorination metal tungsten
}

\author{
Roman V. Brendakov ${ }^{1 *}$, Alexander V. Shvab ${ }^{1}$, and Vladimir N. Brendakov ${ }^{2}$ \\ ${ }^{1}$ Tomsk State University, 634030 Tomsk, Russia \\ ${ }^{2}$ Seversk Technological Institute NRNU MEPhl, 636070 Seversk, Russia
}

\begin{abstract}
In this paper, we consider the process of obtaining the tungsten hexafluoride gas. On the basis of a simple one-component diffusion model of the process of fluorination of tungsten metal recorded an equation relating the rate of a chemical reaction and change in concentration of a key component of the length of the reaction zone. The numerical calculations showed good agreement with experimental data. The statistical treatment of experimental data, constructed regression model for the reaction speed parameter fluoridation as a function that depends on the temperature of the supplied gas and tungsten metal particle size. The results can be used to optimize the design of existing devices and new designs for the fluorination process of metal tungsten.
\end{abstract}

\section{Problem statement}

Process of fluorination of a metal tungsten is carried out according to the chemical reaction presented by a formula

$$
W+3 F_{2}=W F_{6}
$$

To realize fluorination process, gaseous fluorine is passed over a layer of metal tungsten. Passing through the active zone of the reactor, fluorine interacts with tungsten powder to form tungsten hexafluoride $\left(W F_{6}\right)$. Figure 1 shows a schematic diagram of the reaction zone.

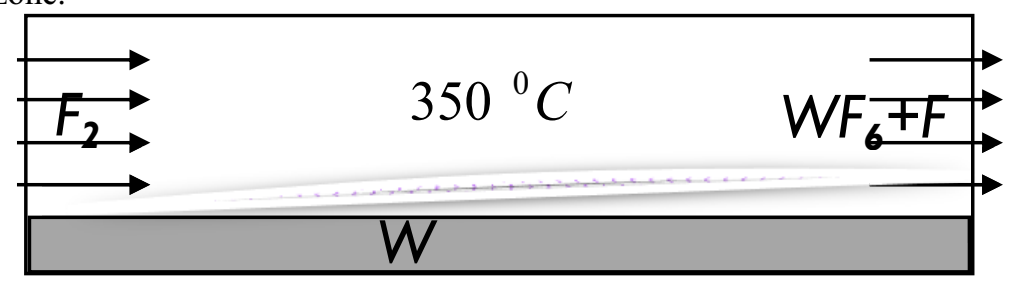

Fig. 1. Scheme of the reaction zone.

As a result of such reaction, concentration of fluorine in a gas stream gradually decreases due to decrease of amount of fluorine and a dilution by its formed $W F_{6}$. Decrease

*Corresponding author: vnbrendakov@mephi.ru 
of concentration of fluorine in bipropellant mix leads reaction rate of fluorination to decrease. At the same time, according to stoichiometric coefficient equal 3, there is a decrease of speed of a gas stream.

In work it is modelled steady-state conditions bipropellant mix of gases of a onedimensional current. The mathematical description of the considered task can be submitted by the equation of one-component diffusion model and the corresponding boundary conditions:

$$
\frac{1}{P e} \frac{d^{2} C(x)}{d x^{2}}=\left(1-\frac{2}{3} C(x)\right) \frac{d C(x)}{d x}-k \cdot(1-C(x))
$$

where $P e=U_{0} H / D$ - number of Peclet, $C=C(x)$ - concentration of a key component (tungsten hexafluoride), $k$-a specific reaction rate.

In work [1] the formula for calculation of reaction rate of fluorination is offered:

$$
V=k \cdot C_{F}
$$

where $C_{F}$ - concentration of gaseous fluorine on the surface of reaction of fluorination.

\section{Numerical calculations}

As practice shows, the specific reaction rate of $\mathrm{k}$ keeps value only under the given conditions. For example, in work [2] it is directly told "Through a difference between temperature on the reacting surface here and temperature of a gas stream expressed reaction rate of fluorination of a tungsten. Temperature difference of subjects is more, than reaction rate is more". And in work [3] the table on dependence of value of a constant of reaction of fluorination on temperature is provided in a zone of reaction $(T)$ and distribution of sizes $(\delta)$ of powder of a metal tungsten. Our regression analysis of the available experimental data allowed to build a functional dependence of the reaction rate constants for fluoridation:

$$
k(\delta, T)=180 \cdot \delta^{(-0,073)} \cdot \mathrm{e}^{(-3970 / T)} .
$$

For check of adequacy of the developed engineering computational method of process of fluorination of a metal tungsten calculations at value of the constants $P e$ were carried out $=1.0$, by $k=0.14$ and comparison of the theoretical graph of concentration of a key component from the longitudinal coordinate and the experimental data from works is executed $[4,5]$.

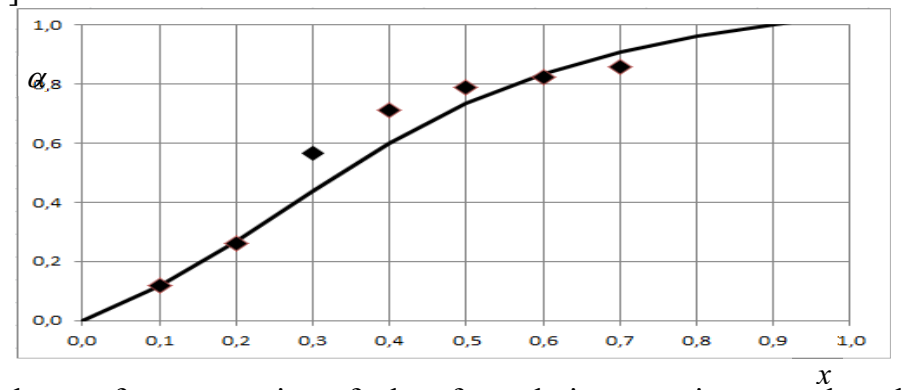

Fig. 2. Dependence of concentration of a key formulation constituent on length reaction zones, solid line - calculation for model, $\boldsymbol{\Delta}$ - data of works [4, 5]. 


\section{Conclusions}

Reasonably good agreement between the calculation and experimental data leads to the conclusion of the performance created engineering calculation method. This approach to the calculation of tungsten fluorination process will carry out rapid assessment of options for the optimal conditions of the process of fluorination of refractory metal powders.

\section{References}

1. Yu. M. Korolev, V. I. Stolyarov, Restoration of fluorides of refractory metals hydrogen (Metallurgy, Moscow, 1981)

2. Patent No. 2310608 - Way of receiving hexafluoride of tungsten. Authors: A. A. Galatian, A. G. Krupin, S. A. Kuzminykh, V. V. Lazarchuk, E. P. Marinenko, A. I. Mines., V. A. Khokhlov (Date of the publication 11/20/2007)

3. Yu. M. Korolev, International Symposium on Inorganic fluorides: Chemistry and Technology (TPU Publishing, Tomsk, 2014)

4. T. Sh. Agnokov, Yu. M. Korolev, M. F. Svidersky, Nalchik: KBGU, 4, 22 (1978)

5. T. Sh. Agnokov, Yu. M. Korolev, M. F. Svidersky, Nalchik: KBGU, 18 (1979) 